\title{
A Schizophrenia Risk Gene, ZNF804A, Influences Neuroanatomical and Neurocognitive Phenotypes
}

\author{
Todd Lencz*,1,2,3, Philip R Szeszko' ${ }^{1,2,3}$, Pamela DeRosse ${ }^{2}$, Katherine E Burdick ${ }^{1,2,3}$, Evelyn J Bromet ${ }^{4}$, \\ Robert M Bilder ${ }^{5}$ and Anil K Malhotra ${ }^{1,2,3}$
}

'Department of Psychiatry, Division of Research, The Zucker Hillside Hospital Division of the North Shore-Long Island Jewish Health System, Glen Oaks, NY, USA; ${ }^{2}$ Center of Psychiatric Neuroscience, The Feinstein Institute for Medical Research, Manhasset, NY, USA; ${ }^{3}$ Department of Psychiatry and Behavioral Science, Albert Einstein College of Medicine of Yeshiva University, Bronx, NY, USA; ${ }^{4}$ Department of Psychiatry, SUNY Stony Brook School of Medicine, Stony Brook, NY, USA; ${ }^{5}$ Department of Psychiatry and Behavioral Sciences, David Geffen School of Medicine, UCLA, Los Angeles, CA, USA

\begin{abstract}
ZNF804A is one of the strongest candidate genes for schizophrenia (SZ), yet its function and role in disease pathophysiology are largely unknown. The only in vivo endophenotype study of the SZ-associated SNP (rs/344706) pointed towards effects on brain functional connectivity. We examined the relationship of this SNP to neuroanatomical and neurocognitive phenotypes that were assessed in healthy individuals. Volunteers with no history of psychiatric illness were assessed with structural magnetic resonance imaging ( I.5T GE scanner, standard gradient-echo acquisition). Carriers of the minor allele were compared with homozygotes for the $T$ (SZ-associated) allele on measures of total volume of the white matter (WM), gray matter (GM), and cerebrospinal fluid compartments, as well as on voxel-wise measurements of regional brain volumes. After examining the correlation between genotype-associated regions of interest and neurocognitive performance measures, the effects of rs I 344706 genotype on a measure of visuomotor performance speed (trails A) were examined in an independent cohort of volunteers. Among healthy subjects, risk allele homozygotes showed larger total WM volumes than carriers of the other allele. Controlling for WM volumes, these same subjects showed reduced GM volumes in several regions comprising the 'default mode network,' including angular gyrus, parahippocampal gyrus, posterior cingulate, and medial orbitofrontal gyrus/gyrus rectus (FDR-corrected $p<0.05$ ). The risk allele dosage also predicted impairments on a timed visuomotor performance task (trails A). Results support a role of ZNF804A in phenotypes reflecting altered neural connectivity.

Neuropsychopharmacology (2010) 35, 2284-229I; doi:I0.1038/npp.2010.102; published online 2I July 2010
\end{abstract}

Keywords: schizophrenia; genetics; endophenotype; ZNF804A; dysconnectivity; default mode network

\section{INTRODUCTION}

Although schizophrenia (SZ) has consistently showed high heritability and elevated sibling recurrence (Sullivan et al, 2003), the identification of susceptibility genes for SZ has proven extremely challenging. However, a recent genomewide association study (GWAS) identified a novel SNP in ZNF804A (rs1344706) as a risk factor for SZ (O'Donovan et al, 2008). In the original report, the association was replicated by pooling multiple cohorts from around the world using meta-analysis. Although many of the individual cohorts yielded statistically non-significant results, the pooled analysis confirmed that the major allele ( $\mathrm{T}$ on the

*Correspondence: Dr T Lencz, Department of Psychiatry, Division of Research, The Zucker Hillside Hospital Division of the North Shore-Long Island Jewish Health System, Psychiatry Research, 75-59 263rd Street, Glen Oaks, NY I 1004, USA, Tel: + I 718470 8126, Fax: + I 7|8343 1659, E-mail: lencz@lij.edu

Received 21 December 2009; revised 7 May 2010; accepted I June 2010 negative strand) was associated with increased risk for SZ. Similar results were obtained in a second GWAS of several independent cohorts from Europe (International Schizophrenia Consortium, 2009), and a combined analysis of $\sim 60000$ individuals confirmed significant $\left(p=4.1 \times 10^{-13}\right)$ association with $\mathrm{SZ}$ and bipolar disorder (Williams et al, 2010).

There is a paucity of data on the function of ZNF804A, a moderately large $(341 \mathrm{~kb})$ gene located on chromosome $2 \mathrm{q} 32.1$. In humans, $Z N F 804 A$ is expressed broadly throughout the brain, especially in the developing hippocampus and the cortex, as well as in the adult cerebellum (Johnson et al, 2009). The functional effects of the risk variant rs1344706, an intronic SNP near the $3^{\prime}$ end of the gene, are also poorly understood. To date, only one laboratory has examined the effects of variation of rs1344706 on any in vivo phenotypes other than DSM-IV-defined psychiatric diagnosis. In two functional magnetic resonance imaging (fMRI) studies of healthy volunteers, allelic variation at this SNP was associated with abnormal patterns of neural connectivity 
across multiple brain areas (Esslinger et al, 2009; Walter et al, 2010), and reduced activation in frontal, temporal/ parietal, and cingulate regions (Walter et al, 2010). Volumetric measures of neuroanatomy were not reported, and the authors suggested that future studies should focus on the effects of rs1344706 variation on measures of brain WM. To date, no studies have investigated effects on performance of standard neurocognitive tests.

Therefore, to further functionally characterize this SNP, we tested the association of rs1344706 with brain structure and neurocognitive performance in healthy individuals. Because biological phenotypes (eg, brain structure and function) are thought to more closely reflect the effects of genetic variation as compared with manifest psychiatric illness, endophenotype studies have proven to be more robust and require vastly smaller sample sizes than purely diagnosis-based studies (Glahn et al, 2007). After first confirming that our allelic frequencies are consistent with the data from previous GWAS, we used structural MRI to determine whether neuroanatomical phenotypes correlate with rs1344706 genotype in healthy individuals. On the basis of the limited previous evidence suggesting widespread expression of ZNF804A in brain and a possible role for rs1344706 in neural dysconnectivity, we predicted widespread effects of allelic variation in cerebral WM and GM. Consequently, we used a multi-stage analytical strategy: first, we examined the total segmented cerebral volumes to test global effects of rs1344706 alleles; next, we used voxel-wise mapping to test the regional anatomical effects of genotype. We then used results of the neuroanatomical study to guide the examination of cognitive correlates of rs1344706 in an independent cohort of healthy individuals.

\section{PARTICIPANTS AND METHODS}

\section{Participants}

Participants were healthy individuals who agreed to participate in neuroimaging $(n=39 ; 19$ male/20 female; mean age $=34.4 \pm 11.9$, range: $18-57$ ) and/or neurocognitive $(n=169 ; 69$ male/100 female; mean age $=40.8 \pm 13.6$, range: 17-65) studies. All subjects also participated as healthy controls in a larger GWAS study of SZ (for additional details, see Supplementary file). Healthy volunteers were recruited from the general population of Queens and Nassau counties on Long Island via word of mouth, newspaper, and internet advertisements, and posted flyers. All participants provided written informed consent to a protocol approved by the Institutional Review Board of the North Shore-Long Island Jewish Health System. Subjects were administered the Structured Clinical Interview for DSM-IV, non-patient version (First et al, 1995). Subjects were excluded if they had a DSM-IV Axis I diagnosis, active or recent substance abuse, or if they had a first degree relative with a known or suspected Axis I disorder, on the basis of family history questionnaire. All potential subjects were screened to rule out history of CNS trauma, neurological disorder, or previously diagnosed learning disability. All subjects were self-reported Caucasians (additional details on ethnicity and tests of population stratification provided in Supplementary file).

\section{DNA Procedures}

Genomic DNA was extracted from whole-blood samples and processed in accordance with the NSLIJHS Institutional Review Board approved Standard Operating Procedures of the Zucker Hillside Hospital DNA Data Bank. Genotyping of rs1344706 was performed as part of a microarray GWAS study (Lencz et al, 2007; Supplementary file). Genotype calls were made using Bayesian Robust Linear Model with Mahalanobis distance classifier algorithm threshold at 0.5 applied to batches of 100 samples. Overall call rate for rs1344706 was $99.1 \%$.

\section{Brain Imaging Acquisition}

MR imaging exams were conducted at the Long Island Jewish Medical Center in the coronal plane using a 3D Fast SPGR with IR Prep $(\mathrm{TR}=12.7$ or $14.7, \mathrm{TE}=4.5$ or $5.5 \mathrm{~ms}$, $\mathrm{FOV}=22 \mathrm{~cm})$ on a $1.5 \mathrm{~T}$ whole-body superconducting imaging system (General Electric, Milwaukee, WI). This sequence produced 124 contiguous images (slice thickness $=1.5 \mathrm{~mm}$ ) of the whole head with nominal in-plane resolution of $0.86 \mathrm{~mm} \times 0.86 \mathrm{~mm}$ in a $256 \times 256$ matrix. Because of one change in software and one change in hardware during the course of the study, scanner platform was added as a three-level factor to all analyses. G-allele carriers were collapsed into a single group $(n=21)$ to provide a more robust sample size for comparisons with T-allele homozygotes $(n=18)$. This grouping was necessary to obtain sufficient power to detect effect sizes of $<1 \mathrm{SD}$, which is approximately the effect size we have observed in a previous imaging genomics study of BDNF (Szeszko et al, 2005). The present study had $70-80 \%$ power to detect Cohen's $d$ of $0.8-0.9$. In addition, follow-up analyses were performed in which each of the three genotype groups were entered separately, to confirm that the two-group results accurately reflected the main effects of rs1344706. Genotype groups were scanned evenly across the three platforms $\left(\chi^{2}(2)=2.57, p=0.28\right)$, as were the two sexes $\left(\chi^{2}(2)=0.78\right.$, $p=0.68)$; moreover, genotype groups did not differ on sex distribution $\left(\chi^{2}(1)=0.02, p=0.88\right)$ or age $\left(t_{37}=0.67\right.$, $p=0.51)$. Axial proton density and T2-weighted images were also obtained to exclude visually detectable structural abnormalities on MR imaging scans.

\section{Brain Imaging Analysis}

Image analysis was performed on a Linux workstation using MATLAB 6.5.1 and SPM5 software (Wellcome Department of Cognitive Neurology, London), with default settings (Rosario et al, 2008). MR images for each subject were spatially normalized from native space to the MNI 152image template, using a 12-parameter affine transformation with 16 non-linear iterations and a $25 \mathrm{~mm}$ non-linear basis function cutoff. Normalized images were then segmented using signal intensity and previous probability information. Intensity modulation was performed on the normalized, segmented MR images by increasing or reducing the voxel intensity depending on whether the surrounding voxels were compressed or expanded in the normalization process (Good et al, 2001). Estimates of total brain size and of brain tissue compartments for whole-brain comparisons were 
obtained automatically from the segmented volumes. Total volumes across genotype groups were compared using MANCOVA, with the total GM, WM, and cerebrospinal volumes entered as dependent measures. Age was entered as a covariate, with sex and scanner platform entered as nuisance factors.

Following whole-brain analysis, the normalized, segmented images were then smoothed with a $12 \mathrm{~mm}$ isotropic Gaussian before performing voxel-wise group comparisons. Group comparisons were performed using ANCOVA in SPM5, with genotype group as a fixed factor. Sex and age were also included in the model. Total intracranial volume was included as an additional covariate for analyses of segmented WM volumes, and the total WM volume was included as a covariate for analyses of segmented GM volumes. The threshold for statistical significance was set to an FDR-corrected $p<0.05$, which has been shown to conservatively protect against Type 1 error (Meyer-Lindenberg et al, 2008), with an additional requirement of a minimum of 100 contiguous above-threshold voxels in a region.

\section{Neurocognitive Testing}

Subjects were administered a brief battery designed to rapidly $(<1 \mathrm{~h})$ obtain measurements across the major domains of neurocognitive function (Burdick et al, 2006). The battery included the following measures: estimated global intellectual functioning (WRAT-3); timed visuomotor performance (trail making test, parts A and B); visual attention (continuous performance test-identical Pairs version); verbal working memory (WAIS-R Digit Span); and verbal learning (California verbal learning test) and fluency (controlled oral word association test).

\section{Statistical Plan}

Brain imaging data analysis is described above and consists of two main tests in healthy subjects: (1) genotype effects (T-allele homozygotes $v s$ G-allele carriers) on whole-brain volumes were tested in MANCOVA with GM volumes, WM volumes, and cerebrospinal fluid volumes entered as dependent measures, and age, sex, and scanner platform entered as covariates; (2) genotype effects on regional volumes at each voxel were tested using ANCOVA, controlling for age, sex, scanner platform, and total intracranial (for regional tests of WM) and WM volumes (for regional tests of GM). In the subset of subjects with both MRI and neurocognitive data $(n=31)$, non-parametric correlational tests (Spearman's rho) were used to identify the cognitive correlates of brain imaging regions of interest associated with rs1344706. Effects of rs1344706 on cognitive measures thus identified were then tested using linear regression, with age as a covariate in the remaining, independent cohort of subjects with neurocognitive data.

\section{RESULTS}

\section{rs1344706 Allele Frequencies}

Allele frequencies almost precisely matched those in the original GWAS sample and in the CEU HapMap sample.
Specifically, overall $\mathrm{T}$-allele frequency in the present study was $59.3 \%$ in controls; $\mathrm{T}$-allele frequency was $59 \%$ among 2937 Caucasian controls in the original GWAS discovery sample and is $57 \%$ in the HapMap CEU cohort.

\section{Association with Structural MRI Volumes in Healthy Subjects}

Total WM, GM, and cerebrospinal fluid volumes were entered into a MANCOVA, with age, sex, and scanner parameters as covariates. The multivariate analysis showed a significant effect of rs1344706 on the brain volume measures $(\mathrm{F} 3,25=6.76, p=0.002)$. Consistent with the dysconnectivity hypothesis, univariate tests of between-subjects effects showed that the effect of rs1344706 genotype on total brain volumes was specific to WM. Specifically, T-allele homozygotes showed large total WM volumes $(\mathrm{F} 1,27=4.61$, $p=0.041$, partial eta $\left.{ }^{2}=0.146\right)$ compared with non-homozygotes. Genotype groups did not significantly differ in total GM $(\mathrm{F} 1,27=0.13, p=0.722)$ or cerebrospinal fluid volumes $(\mathrm{F} 1,27=1.15, p=0.294$; Figure 1). Results did not substantially change when each of the three genotype groups was examined separately. As shown in Figure 2, the T-allele homozygotes differed in WM volume from both G-allele homozygotes and heterozygotes, which were nearly identical in volume. Genotype effects on GM and cerebrospinal fluid volumes remained non-significant.

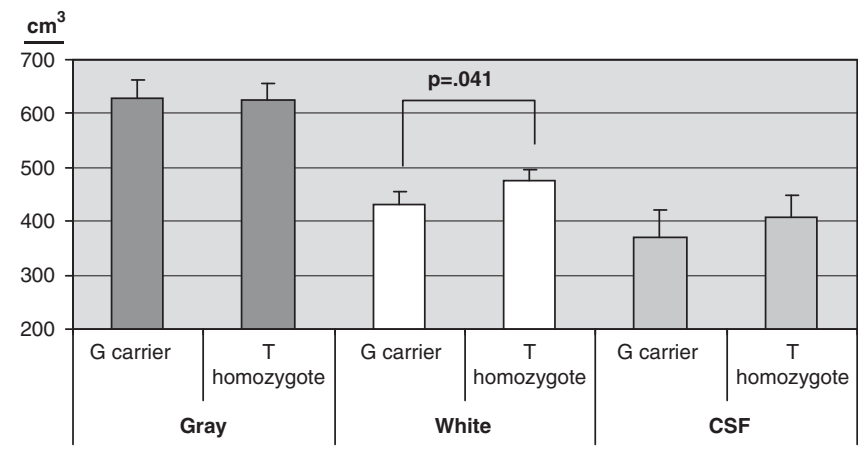

Figure I Covariate-corrected, segmented volumes for GM, WM, and CSF compartments in healthy subjects divided by rs 344706 genotype (G-allele carriers, $n=21$ vs T-allele homozygotes, $n=18$ ). Error bars represent twice the SE of the mean.

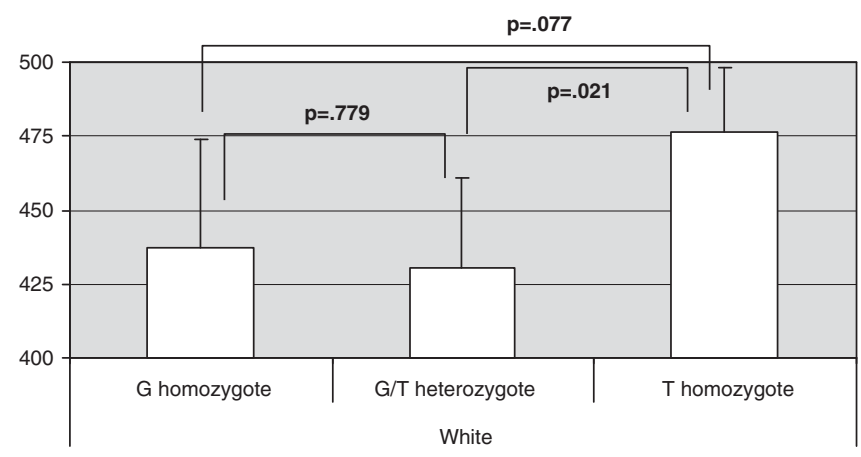

Figure 2 Covariate-corrected, segmented volumes for WM in healthy subjects divided by rs 1344706 genotype (G-allele homozygotes, $n=7$; G/T heterozygotes, $n=14$; T-allele homozygotes, $n=18$ ). Error bars represent twice the SE of the mean. 
Table I Gray Matter Regions Significantly Smaller in Healthy Volunteers Homozygous for the T-allele at rs 344706 Compared with Healthy G-allele Carriers (FDR-Corrected $p<0.05$; Minimum of 100 Contiguous Voxels)

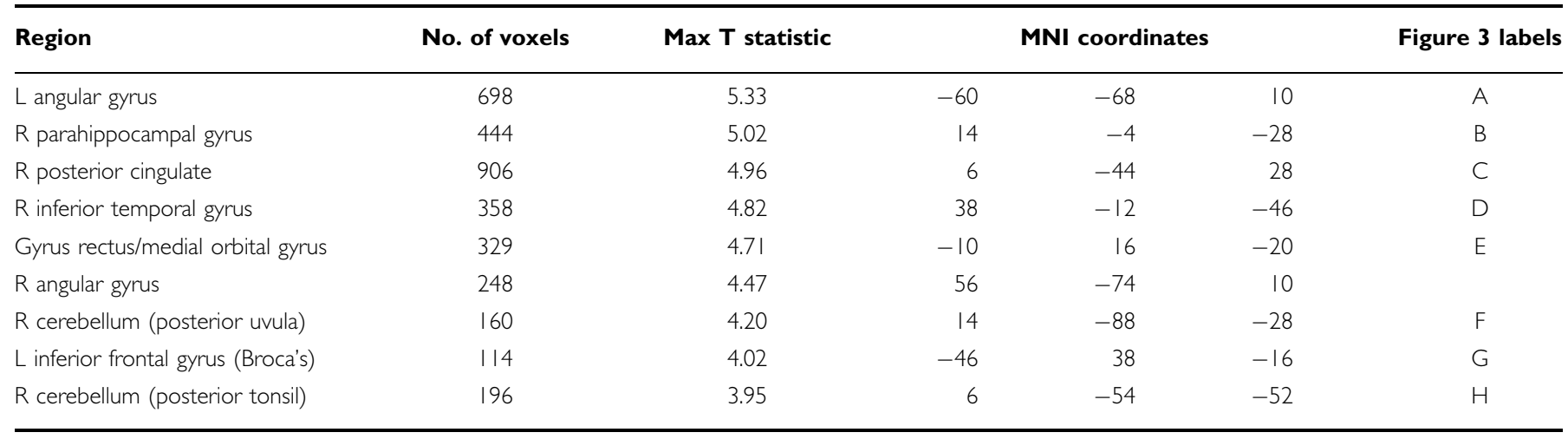

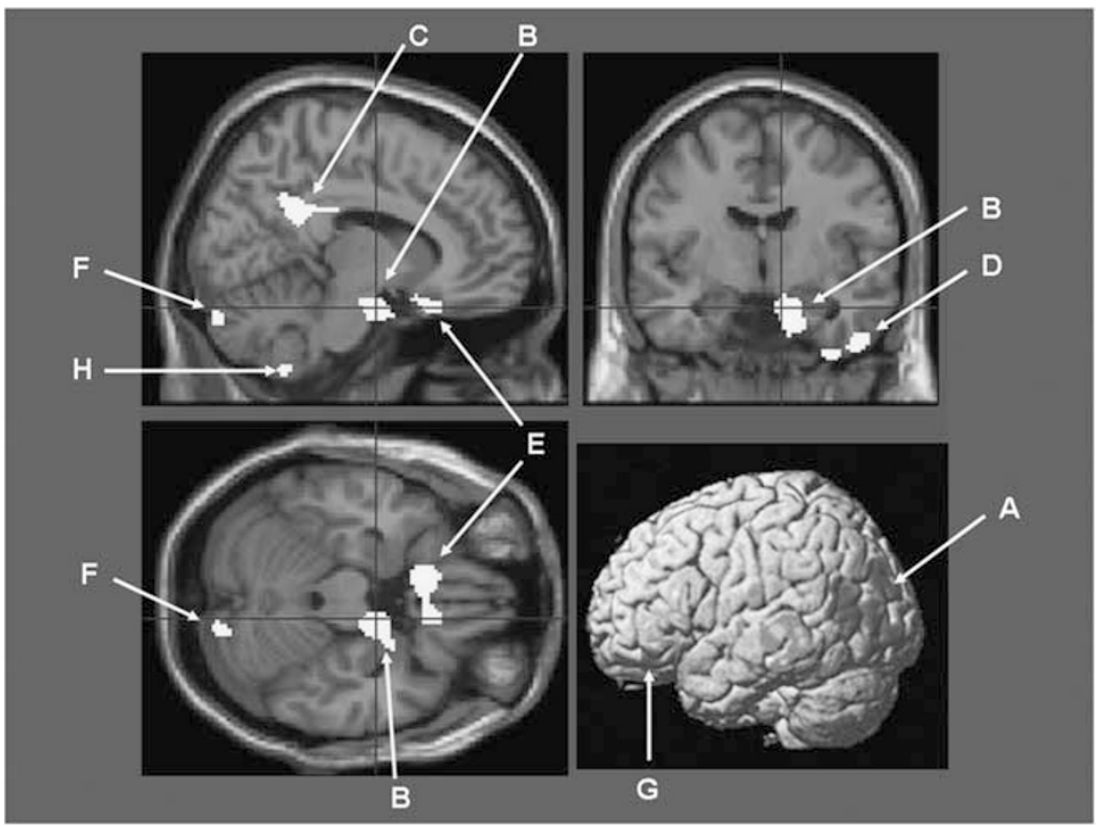

Figure 3 Visualization of GM regions, which are significantly smaller in healthy volunteers homozygous for the T-allele at $r s$ I 344706 ( $n=\mid 8)$ compared with healthy G-allele carriers ( $n=21$; FDR-corrected $p<0.05$; minimum of 100 contiguous voxels). All images depicted on the canonical MNI template provide in SPM5. Legends for region labels A-H are provided in the right-most column of Table I.

In voxel-wise analysis using SPM5, the effect of genotype on WM volume was distributed evenly across the entire WM compartment as a single region (data not shown). The fact that total GM volumes did not differ between genotype groups, despite significantly larger WM volumes, suggested the possibility of regionally localized GM deficits in T-allele homozygotes. Therefore, we tested genotypic effects on regional GM volumes, controlling for the total WM volume (and the aforementioned covariates). On applying a strict threshold for statistical significance, which has been shown to conservatively control the Type 1 error (FDR-corrected $p<0.05$; Meyer-Lindenberg et al, 2008), we observed nine regions (minimum extent $=100$ voxels) that were significantly smaller in subjects who were homozygous for the risk (T) allele compared with non-risk (G) allele carriers (Table 1; Figure 3). These regions included multiple components of the 'default mode network' (Raichle et al, 2001), including parahippocampal gyrus, posterior cingulate, ventromedial prefrontal cortex, and lateral parietal cortex (angular gyrus). No regions were significantly (FDR-corrected $p<0.05$ ) larger in T-allele homozygotes compared with G-allele carriers.

\section{Brain Structure-Function Relationships}

Standardized residual values from the peak voxel of each of the nine regions listed in Table 1 were averaged, yielding a single measure of rs1344706-associated GM for each subject. Correlations between this composite score and the dependent measures from the neurocognitive battery were examined using Spearman's rho. Nominally significant correlations were obtained for trails A $(\rho=-0.36$, $p=0.048$ ), indicating that reduced GM volumes across the 


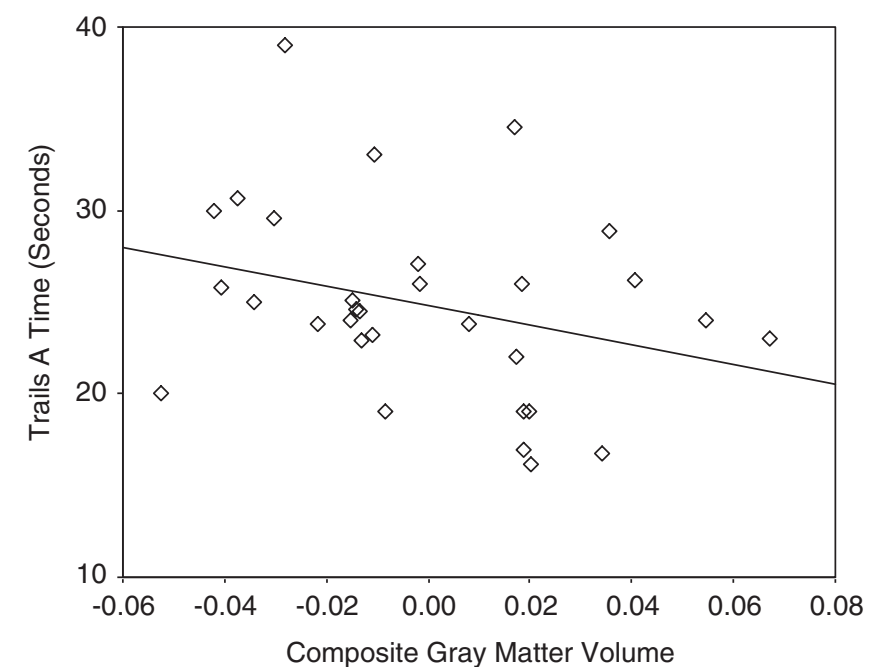

Figure 4 Relationship between composite regional GM volume (expressed as a standardized metric) and performance on trail making test, part $A(n=3 \mid$ subjects with both measures available). Note that longer times indicate worse performance.

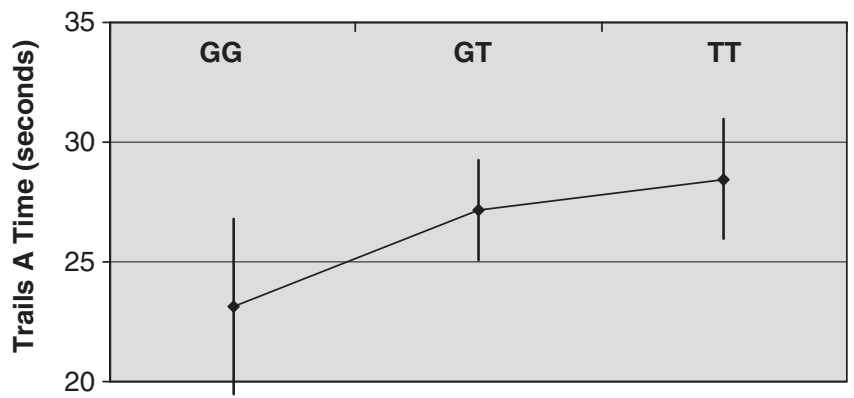

Figure 5 Relationship between rs/344706 genotype (G-allele homozygotes, $n=22$; $G / T$ heterozygotes, $n=67$; T-allele homozygotes, $n=47$ ) and performance (time to completion) on trails $A$ in healthy volunteers.

nine implicated regions were associated with longer times (poorer performance) on this test (Figure 4). By contrast, there was no significant correlation between the GM index and trails B performance, after correction for trails A performance $(\rho=0.091, p=0.65)$. The absolute value of the correlations with other neurocognitive measures were all $<0.1$, and all $p$-values were $>0.58$.

\section{Association With Neurocognitive Performance in Healthy Subjects}

We next examined the effects of rs1344706 on trails A in the remaining cohort (ie, subjects without MRI data, who were therefore, not part of the analyses described immediately above). As shown in Figure 5, there was a linear relationship between $\mathrm{T}$-allele dosage and poorer performance, as indexed by longer times to complete the task. Linear regression, after controlling for the effects of age, indicated that this relationship was statistically significant, with allele dosage accounting for $3.5 \%$ of the variance in trails $\mathrm{A}$ times $(\beta=0.188, t=2.25, \quad p=0.026)$. Results were essentially identical when skewness of the dependent measure was reduced using log-transformation or square-root transformation, and when estimated IQ was added as a covariate. Slightly stronger associations between rs1344706 T-allele dosage and trails A performance were observed when the subjects with MRI data were also included in the analysis ( $\beta=0.190, t=2.52, p=0.013)$. No significant associations were observed to other neurocognitive domains in our battery (all $p$ 's $>0.12$ ). Although trails A was selected for investigation a priori on the basis of its correlation with MRI volumes, it should be noted that the effect on trails A was modest and would only reach marginal significance $(p=0.065)$ if strict Bonferroni correction were applied against all five domain scores in our neurocognitive battery.

\section{DISCUSSION}

To our knowledge, this is the first study to identify neuroanatomical and neurocognitive phenotypes associated with the rs1344706 polymorphism in ZNF804A. This SNP has emerged as an important risk candidate for SZ, with convergent support across two large GWAS studies (O'Donovan et al, 2008; International Schizophrenia Consortium, 2009) and independent replication samples (Riley et al, 2010; Williams et al, 2010), including our small case-control study (Supplementary text). By examining intermediate phenotypes, our study provides evidence regarding the possible pathophysiological mechanisms underlying this susceptibility.

Consistent with a previous fMRI study (Esslinger et al, 2009), our data support the hypothesis that ZNF804A variation influences patterns of neural connectivity across a distributed network of brain regions, as evidenced by the altered WM volumes distributed across the entire brain. Although the direction of WM differences (greater volume in risk allele homozygotes) was unanticipated, it is consistent with a recent bioinformatic analysis (Riley et al, 2010): in silico analysis predicted that the risk allele yields increased binding sites for two transcription factors implicated in the proliferation of oligodendrocytes. In our study, the association of genetic risk with increased WM volume is also consistent with previous reports of increased WM volume in first-degree relatives of patients with SZ (Marcelis et al, 2003), and individuals with schizotypal personality disorder (Hazlett et al, 2008). Studies of WM volumes in patients with SZ have yielded mixed results (Honea et al, 2005); although several studies have observed reduced WM volumes in SZ, these deficits may be highly focal (Di et al, 2009; Ueda et al, 2010). Global reductions in WM that are sometimes observed in SZ may be a function of differential aging and medication effects (Bose et al, 2009; Bartzokis et al, 2009), and are not observed in first-episode patients (Crespo-Facorro et al, 2009; Moriya et al, 2010).

To our knowledge, this is also the first study to identify a genetic correlate of multiple brain regional GM volumes comprising the default mode network. Previous studies in the imaging genetics literature have focused on specific regional volumetrics, such as hippocampus (Szeszko et al, 2005), or on specific localized circuits such as frontolimbic pathways (Pacheco et al, 2009). Notably, several regions in which the risk allele is associated with reduced GM volumes in our analysis also show reduced activation in a fMRI study (Walter et al, 2010). These regions include left angular 
gyrus, left inferior frontal gyrus, and posterior cingulate, all of which comprise a part of the default mode network (Raichle et al, 2001).

The default mode network comprises regions that show synchronized activity at 'resting' baseline, in the absence of specific stimulation (Raichle et al, 2001). Ongoing work has implicated this network in the development of selfreferential thought (Spreng and Grady, 2010), which has been specifically implicated in the developmental psychopathology of SZ (Nelson et al, 2009). Aberrant activity and functional connectivity across the default network regions (including posterior cingulate, parahippocampal gyrus, parietal cortex, and medial prefrontal cortex) have been repeatedly observed in patients with SZ (Kim et al, 2009), and is similarly observed in the first-degree relatives of SZ patients (Whitfield-Gabrieli et al, 2009). Structural abnormalities of these regions, as indexed by volumetric deficits, have also been shown to be heritable components of SZ in MRI studies of first-degree relatives (Seidman et al, 2003; Goldman et al, 2009). Notably, the connectivity of this network is not observed in pre-adolescent children (Fair et al, 2008), and its development is thought to coincide with the adolescent/early adulthood time period during which SZ onset typically occurs (Nelson et al, 2009). Thus, ZNF804A variation may have a role in this SZ-related neurodevelopmental abnormality.

In our sample of healthy and cognitively normal individuals, the risk allele seems to impact timed neuromotor performance, as indexed by the trail making test (trails A). Neuroimaging studies have previously shown that visuomotor processing speed depends on WM integrity across large regions of the brain (Aukema et al, 2009; van Swieten et al, 1996), and is more sensitive than many other cognitive assays to age-related decrements in widespread GM volumes (Coffey et al, 2001). Our observation that trails A performance was significantly correlated with regional GM volumes is consistent with this literature.

An important methodological consideration is that effect sizes for biological phenotypes, which may be more proximal to the effects of genetic variation at the molecular level, may be much larger than genetic effect sizes for psychiatric diagnosis. For example, in our study, variation at rs1344706 accounted for approximately 15\% of the variation in total WM volume (after controlling for covariates). This is comparable to our previous study of $B D N F$ genetic effects on hippocampal volumes, in which a single polymorphism accounted for $>20 \%$ of the volumetric variance (Szeszko et al, 2005); this large effect size was also observed by independent groups studying the relationship of $B D N F$ to hippocampal volumes (Pezawas et al, 2004; Bueller et al, 2006; Frodl et al, 2007). Other results in the present study followed a predicted, descending order of effect sizes across phenotypes. In the neurocognitive analysis, T-allele dosage accounted for $3.5 \%$ of the variance in trails A performance. By contrast, rs 1344706 effects accounted for $<1 \%$ of the variance in SZ diagnosis, consistent with previous studies in psychiatric genetics (O'Donovan et al, 2008; International Schizophrenia Consortium, 2009; Riley et al, 2010; Allen et al, 2008). Other phenotypes not examined in the present study (eg, gene expression, Riley et al, 2010) may be even more robust targets for analysis.
These large effect sizes for biological variables are counterbalanced by the fact that studies of extensive biological and clinical phenotypes cannot readily generate large sample sizes of the sort that are now common in GWAS studies of diagnostic categories. Our study used relatively small samples of healthy controls to examine effects on brain imaging and neurocognitive performance, and it is our hope that this report will stimulate the accumulation of additional data sets for future metaanalysis. This limitation of our study was partially offset by: (1) the usage of large-scale published GWAS data as a starting point for candidate gene identification and (2) the fact that a second, independent cohort was available for testing the neurocognitive phenotype that was associated with brain volumes in the initial MRI cohort. Nevertheless, these data reinforce the importance of efforts to collect large samples of healthy controls characterized across a range of brain structural and functional phenotypes. In particular, the present study and the previous fMRI study (Esslinger et al, 2009), suggest that WM integrity, as measured by diffusion tensor imaging, may be an important endophenotype for future investigations of this gene.

\section{ACKNOWLEDGEMENTS}

This work was supported by the Donald and Barbara Zucker Foundation, internal funding from the North Shore-Long Island Jewish Health System, and Grants from the Stanley Foundation (to EJB), the National Alliance for Research on Schizophrenia and Depression (to AKM), and the National Institutes of Health (K23MH001760 and R01MH079800 to AKM; R01MH0084098 to TL; R01MH044801 to EJB; R01MH060374 to RMB; and Center Grants P30MH074543 to John M Kane and M01RR018535 to Kevin J Tracey).

\section{DISCLOSURE}

Financial Disclosure: Dr Lencz has received consulting fees or honoraria from Merck, Eli Lilly \& Company, Cowen \& Co., Guidepoint Global, Golden Helix, InforMed Insights, and PGxHealth (a division of Clinical Data). Dr Bilder has received consulting fees or honoraria from: the Abbott Laboratories, American Psychiatric Association, American Psychiatric Institute for Research and Education, American Psychological Association, Arbor Scientia, Arthur D Little, Biostrategies Group, Braun Consulting, CHDI, Cogtest, Cypress Bioscience, Dainipon Sumitomo, Duke University, ESI Education, Foundation for Psychocultural Research, Healthcare Analytics, Hollis \& Associates, IMEDEX, InforMed, International Neuropsychological Society, Johnson \& Johnson, Lippincott Williams \& Wilkins, Medacorp, Memory Pharmaceuticals, National Institutes of Health, Partners Healthcare System, Pfizer, Philadelphia Neuropsychology Group, Roche, The Help Group, and Vanda Pharmaceuticals. Dr Malhotra has received consulting fees or honoraria from Eli Lilly \& Company, Janssen Pharmaceutica, Merck, Bristol-Meyers Squibb, Pfizer, PGxHealth (a division of Clinical Data), Roche Diagnostics, and Vanda Pharmaceuticals, and has received research support from Eli Lilly \& Company. Drs Szeszko, DeRosse, Burdick, and Bromet do not have financial disclosures to report. 
Disclaimer: The funding agencies did not have a role in the design and conduct of the study; collection, management, analysis, and interpretation of the data; and preparation, review, or approval of the paper.

\section{REFERENCES}

Allen NC, Bagade S, McQueen MB, Ioannidis JP, Kavvoura FK, Khoury MJ et al (2008). Systematic meta-analyses and field synopsis of genetic association studies in schizophrenia: the SzGene database. Nat Genet 40: 827-834.

Aukema EJ, Caan MW, Oudhuis N, Majoie CB, Vos FM, Reneman L et al (2009). White matter fractional anisotropy correlates with speed of processing and motor speed in young childhood cancer survivors. Int J Radiat Oncol Biol Phys 74: 837-843.

Bartzokis G, Lu PH, Stewart SB, Oluwadara B, Lucas AJ, Pantages J et al (2009). In vivo evidence of differential impact of typical and atypical antipsychotics on intracortical myelin in adults with schizophrenia. Schizophr Res 113: 322-331.

Bose SK, Mackinnon T, Mehta MA, Turkheimer FE, Howes OD, Selvaraj S et al (2009). The effect of ageing on grey and white matter reductions in schizophrenia. Schizophr Res 112: 7-13.

Bueller JA, Aftab M, Sen S, Gomez-Hassan D, Burmeister M, Zubieta JK (2006). BDNF Val66Met allele is associated with reduced hippocampal volume in healthy subjects. Biol Psychiatry 59: 812-815.

Burdick KE, Lencz T, Funke B, Finn CT, Szeszko PR, Kane JM et al (2006). Genetic variation in DTNBP1 influences general cognitive ability. Hum Mol Genet 15: 1563-1568.

Coffey CE, Ratcliff G, Saxton JA, Bryan RN, Fried LP, Lucke JF (2001). Cognitive correlates of human brain aging: a quantitative magnetic resonance imaging investigation. J Neuropsychiatry Clin Neurosci 13: 471-485.

Crespo-Facorro B, Roiz-Santiáñez R, Pérez-Iglesias R, TordesillasGutiérrez D, Mata I, Rodríguez-Sánchez JM et al (2009). Specific brain structural abnormalities in first-episode schizophrenia. A comparative study with patients with schizophreniform disorder, non-schizophrenic non-affective psychoses and healthy volunteers. Schizophr Res 115: 191-201.

Di X, Chan RC, Gong QY (2009). White matter reduction in patients with schizophrenia as revealed by voxel-based morphometry: an activation likelihood estimation meta-analysis. Prog Neuropsychopharmacol Biol Psychiatry 33: 1390-1394.

Esslinger C, Walter H, Kirsch P, Erk S, Schnell K, Arnold C et al (2009). Neural mechanisms of a genome-wide supported psychosis variant. Science 324: 605.

Fair DA, Cohen AL, Dosenbach NU, Church JA, Miezin FM, Barch DM et al (2008). The maturing architecture of the brain's default network. Proc Natl Acad Sci USA 105: 4028-4032.

First MB, Spitzer RL, Gibbon M, Williams JBW (1995). Structured Clinical Interview for DSM-IV Axis I Disorders, Non-Patient Edition (SCID-NP). Biometric Research Department: New York.

Frodl T, Schüle C, Schmitt G, Born C, Baghai T, Zill P et al (2007). Association of the brain-derived neurotrophic factor Val66Met polymorphism with reduced hippocampal volumes in major depression. Arch Gen Psychiatry 64: 410-416.

Glahn DC, Thompson PM, Blangero J (2007). Neuroimaging endophenotypes: strategies for finding genes influencing brain structure and function. Hum Brain Mapp 28: 488-501.

Good CD, Johnsrude IS, Ashburner J, Henson RN, Friston KJ, Frackowiak RS (2001). A voxel-based morphometric study of ageing in 465 normal adult human brains. Neuroimage 14: 21-36.

Goldman AL, Pezawas L, Mattay VS, Fischl B, Verchinski BA, Chen Q et al (2009). Widespread reductions of cortical thickness in schizophrenia and spectrum disorders and evidence of heritability. Arch Gen Psychiatry 66: 467-477.
Hazlett EA, Buchsbaum MS, Haznedar MM, Newmark R, Goldstein KE, Zelmanova Y et al (2008). Cortical gray and white matter volume in unmedicated schizotypal and schizophrenia patients. Schizophr Res 101: 111-123.

Honea R, Crow TJ, Passingham D, Mackay CE (2005). Regional deficits in brain volume in schizophrenia: a meta-analysis of voxel-based morphometry studies. Am J Psychiatry 162: 2233-2245.

International Schizophrenia Consortium (2009). Common polygenic variation contributes to risk of schizophrenia and bipolar disorder. Nature 460: 748-752.

Johnson MB, Kawasawa YI, Mason CE, Krsnik Z, Coppola G, danoviæ D et al (2009). Functional and evolutionary insights into human brain development through global transcriptome analysis. Neuron 62: 494-509.

Kim DI, Manoach DS, Mathalon DH, Turner JA, Mannell M, Brown GG et al (2009). Dysregulation of working memory and default-mode networks in schizophrenia using independent component analysis, an fBIRN and MCIC study. Hum Brain Mapp 30: 3795-3811.

Lencz T, Morgan TV, Athanasiou M, Dain B, Reed CR, Kane JM et al (2007). Converging evidence for a pseudoautosomal cytokine receptor gene locus in schizophrenia. Mol Psychiatry 12: $572-580$.

Marcelis M, Suckling J, Woodruff P, Hofman P, Bullmore E, van Os J (2003). Searching for a structural endophenotype in psychosis using computational morphometry. Psychiatry Res 122: 153-167.

Meyer-Lindenberg A, Nicodemus KK, Egan MF, Callicott JH, Mattay V, Weinberger DR (2008). False positives in imaging genetics. Neuroimage 40: 655-661.

Moriya J, Kakeda S, Abe O, Goto N, Yoshimura R, Hori H et al (2010). Gray and white matter volumetric and diffusion tensor imaging (DTI) analyses in the early stage of first-episode schizophrenia. Schizophr Res 116: 196-203.

Nelson B, Fornito A, Harrison BJ, Yücel M, Sass LA, Yung AR et al (2009). A disturbed sense of self in the psychosis prodrome: linking phenomenology and neurobiology. Neurosci Biobehav Rev 33: 807-817.

O’Donovan MC, Craddock N, Norton N, Williams H, Peirce T, Moskvina $\mathrm{V}$ et al (2008). Identification of loci associated with schizophrenia by genome-wide association and follow-up. Nat Genet 40: 1053-1055.

Pacheco J, Beevers CG, Benavides C, McGeary J, Stice E, Schnyer DM (2009). Frontal-limbic white matter pathway associations with the serotonin transporter gene promoter region (5-HTTLPR) polymorphism. J Neurosci 29: 6229-6233.

Pezawas L, Verchinski BA, Mattay VS, Callicott JH, Kolachana BS, Straub RE et al (2004). The brain-derived neurotrophic factor val66met polymorphism and variation in human cortical morphology. J Neurosci 24: 10099-10102.

Raichle ME, MacLeod AM, Snyder AZ, Powers WJ, Gusnard DA, Shulman GL (2001). A default mode of brain function. Proc Natl Acad Sci USA 98: 676-682.

Riley B, Thiselton D, Maher BS, Bigdeli T, Wormley B, McMichael $\mathrm{GO}$ et al (2010). Replication of association between schizophrenia and ZNF804A in the Irish case-control study of sSchizophrenia sample. Mol Psychiatry 15: 29-37.

Rosario BL, Ziolko SK, Weissfeld LA, Price JC (2008). Assessment of parameter settings for SPM5 spatial normalization of structural MRI data: application to type 2 diabetes. Neuroimage 41: 363-370.

Seidman LJ, Pantelis C, Keshavan MS, Faraone SV, Goldstein JM, Horton NJ et al (2003). A review and new report of medial temporal lobe dysfunction as a vulnerability indicator for schizophrenia: a magnetic resonance imaging morphometric family study of the parahippocampal gyrus. Schizophr Bull 29: 803-830.

Spreng RN, Grady CL (2010). Patterns of brain activity supporting autobiographical memory, prospection, and theory-of-mind and 
their relationship to the default mode network. J Cogn Neurosci 22: $1112-1123$.

Sullivan PF, Kendler KS, Neale MC (2003). Schizophrenia as a complex trait: evidence from a meta-analysis of twin studies. Arch Gen Psychiatry 60: 1187-1192.

Szeszko PR, Lipsky R, Mentschel C, Robinson D, Gunduz-Bruce H, Sevy S et al (2005). Brain-derived neurotrophic factor val66met polymorphism and volume of the hippocampal formation. $\mathrm{Mol}$ Psychiatry 10: 631-636.

Ueda K, Fujiwara H, Miyata J, Hirao K, Saze T, Kawata R et al (2010). Investigating association of brain volumes with intracranial capacity in schizophrenia. Neuroimage 49: 2503-2508.

van Swieten JC, Staal S, Kappelle LJ, Derix MM, van Gijn J (1996). Are white matter lesions directly associated with cognitive impairment in patients with lacunar infarcts? J Neurol 243: 196-200.

Walter H, Schnell K, Erk S, Arnold C, Kirsch P, Esslinger C et al (2010). Effects of a genome-wide supported psychosis risk variant on neural activation during a theory-of-mind task. Mol Psychiatry. [Epub ahead of print].

Whitfield-Gabrieli S, Thermenos HW, Milanovic S, Tsuang MT, Faraone SV, McCarley RW et al (2009). Hyperactivity and hyperconnectivity of the default network in schizophrenia and in first-degree relatives of persons with schizophrenia. Proc Natl Acad Sci USA 106: 1279-1284.

Williams HJ, Norton N, Dwyer S, Moskvina V, Nikolov I, Carroll L et al (2010). Fine mapping of ZNF804A and genome-wide significant evidence for its involvement in schizophrenia and bipolar disorder. Mol Psychiatry. [Epub ahead of print].

Supplementary Information accompanies the paper on the Neuropsychopharmacology website (http://www.nature.com/npp) 